\title{
Solitons in a wave tank
}

\section{Olsen, M.; Smith, H.; Scott, Alwyn C.}

\section{Published in:}

American Journal of Physics

Link to article, DOI:

10.1119/1.13542

Publication date:

1984

\section{Document Version}

Publisher's PDF, also known as Version of record

Link back to DTU Orbit

\section{Citation (APA):}

Olsen, M., Smith, H., \& Scott, A. C. (1984). Solitons in a wave tank. American Journal of Physics, 52(9), 826830. https://doi.org/10.1119/1.13542

\section{General rights}

Copyright and moral rights for the publications made accessible in the public portal are retained by the authors and/or other copyright owners and it is a condition of accessing publications that users recognise and abide by the legal requirements associated with these rights.

- Users may download and print one copy of any publication from the public portal for the purpose of private study or research.

- You may not further distribute the material or use it for any profit-making activity or commercial gain

- You may freely distribute the URL identifying the publication in the public portal

If you believe that this document breaches copyright please contact us providing details, and we will remove access to the work immediately and investigate your claim 
spectra illustrate clearly the simple "quasiatomic" and "quasifree electron" models taught at the start of undergraduate solid state lectures.

\section{ACKNOWLEDGMENTS}

The authors are very grateful to $K$. Siegbahn and U. Gelius from Uppsala University (Sweden) where part of the measurements were performed. J. J. P. thanks the Fonds National de la Recherche Scientifique (Belgium) for financial support. a) Research associate of the National Fund for Scientific Research (Belgium).

'J. J. Pireaux, S. Svensson, E. Basilier, P-A. Malmovist, U. Gelius, R. Caudano, and K. Siegbahn, Phys. Rev. A 14, 2133 (1976).

${ }^{2}$ J. J. Pireaux and R. Caudano, Phys. Rev. B 15, 2242 (1977).

${ }^{3}$ e. g., Photoemission in Solids, edited by M. Cardona and L. Ley (SpringerVerlag, Berlin, 1978).

${ }^{4}$ J. Delhalle, J. M. Andre, S. Delhalle, J. J. Pireaux, R. Caudano, and J. Verbist, J. Chem. Phys. 60, 595 (1974).

${ }^{5} \mathrm{C}$. Cohen-Taunnoudji, B. Diu, and F. Laloe, Mécanique Quantique (Hermann, Paris, 1973).

\title{
Solitons in a wave tank
}

\author{
$M$. Olsen and $H$. Smith \\ Physics Laboratory I, H. C. Ørsted Institute, Copenhagen, Denmark \\ A. C. Scotta) \\ Laboratory of Applied Mathematical Physics, Technical University of Denmark, Lyngby, Copenhagen, \\ Denmark
}

(Received 10 August 1983; accepted for publication 4 November 1983)

A wave tank experiment (first described by the nineteenth-century engineer and naval architect John Scott Russell) relates a linear eigenvalue problem from elementary quantum mechanics to a striking feature of modern nonlinear wave theory: multiple generation of solitons. The tank experiment is intended for lecture demonstrations.

\section{INTRODUCTION}

The term "soliton" was coined by Zabusky and Kruskal" in 1965 in connection with computer studies of solitary wave solutions for nonlinear partial differential equations. They showed that certain waves would not destroy themselves upon collision with each other (as was generally expected by applied mathematicians of that day) but would emerge from a collision having exactly the same shapes and speeds with which they entered. Such waves were recognized as more than oddities in the attic of science; they became the primary components in many important problems of nonlinear wave dynamics. Today an understanding of soliton theory is essential for research in solid-state physics; and many other areas of applied science, including plasma physics, optical communications, polymer dynamics, hydrodynamics, the nature of elementary particles, and biochemistry have been profoundly influenced.

The mystery behind the soliton began to unravel in 1967 when Gardner et $a l^{2}$ demonstrated a totally unexpected property of the nonlinear wave equation

$$
\frac{\partial \Phi}{\partial t}-6 \Phi \frac{\partial \Phi}{\partial x}+\frac{\partial^{3} \Phi}{\partial x^{3}}=0
$$

The property is this: If a local solution of $(1.1)$ is taken as the potential function in the Schrödinger equation

$$
\frac{\partial^{2} \psi}{\partial x^{2}}+[E-\Phi(x, t)] \psi=0,
$$

the bound state eigenvalues are independent of time. Moreover, each bound state eigenvalue $E_{n}$ of (1.2) corresponds to a particular soliton component

$$
\widetilde{\Phi}_{n}(x, t)=-\frac{1}{2} v_{n} \operatorname{sech}^{2}\left[\frac{1}{2} \sqrt{v_{n}}\left(x-v_{n} t\right)\right]
$$

in the general solution of (1.1). The correspondence is given by

$$
E_{n}=-v_{n} / 4 \text {. }
$$

In addition to (1.1) a large family of nonlinear wave equations has been discovered to share identical properties and several books have recently been published discussing these developments in detail ${ }^{3-13}$; thus it is now feasible to teach soliton theory in the undergraduate physics curriculum. We have been engaged in this activity for several years and we have found demonstration experiments to be very helpful in convincing the student that soliton mathematics is related to the real world. ${ }^{14}$ The aim of this paper is to describe such a demonstration experiment for Eq. (1.1).

Equation (1.1) was first derived in 1895 by Korteweg and deVries ${ }^{15}$ to describe observations on shallow water waves made by the British engineer and naval architect John Scott Russell ${ }^{16}$ in the decade from 1834 to 1844 . Upon returning to this early work one again encounters a surprise: The most striking aspects of soliton behavior were seen by Russell on a water tank which he described as being " $A$ foot wide, eight or nine inches deep and twenty or thirty feet long." In particular, he notes "The genesis... of a compound or double wave of the first order, which immediately breaks down by spontaneous analysis into two, the greater going faster and altogether leaving the smaller." It is precisely this "spontaneous analysis" that is predicted by 
$(1.1)-(1.4)$ when the Schrödinger equation (1.2) has more than one bound state. ${ }^{17}$ Merely by following Russell's instructions we arrive at the interesting and helpful lecture demonstration that is described here.

In Sec. II we derive the Korteweg-deVries equation for waves of moderate amplitude propagating in one direction in shallow water of uniform depth. We then normalize this equation to the form given in (1.1) and derive in Sec. III the solitary wave solution (1.3). In Sec. IV we discuss the relation of these results to the Schrödinger equation (1.2). Next (Sec. V) we describe a wave tank design that is suitable for lecture demonstration, and finally in Sec. VI we present a variety of experimental results that are readily obtained from measurements on the wave tank.

\section{THE LONG WAVE EQUATION}

The long wave or Korteweg-deVries equation describes waves of small but finite amplitude propagating in one direction in shallow water. In this section we summarize the physical assumptions underlying the Korteweg-deVries equation and give the main steps in its derivation. For details the reader may consult the book by Whitham. ${ }^{18}$

We assume the liquid to be incompressible and frictionless with an irrotational velocity field $\mathbf{v}$ satisfying $\operatorname{curl} \mathbf{v}=0$. The resulting potential flow is given by $v=\operatorname{grad} \phi$, where $\phi$ is the velocity potential. For incompressible liquids the continuity equation takes the form

$\operatorname{div} \mathbf{v}=0$

which in connection with $\mathbf{v}=\operatorname{grad} \phi$ yields the Laplace equation

$$
\Delta \phi=0 \text {, }
$$

which must be satisfied by the velocity potential everywhere in the bulk.

Let us consider waves propagating in the $x$ direction in a water tank of depth $h$. We need to specify the boundary conditions at the bottom of the $\operatorname{tank}(z=-h)$ as well as at the free surface. In equilibrium $z=0$ at the free surface. When waves propagate, the position of the surface is specified by the function $u(x, t)$ defined by

$$
z=u(x, t)
$$

at the free surface. At the bottom of the tank the velocity must be zero in the $z$ direction, but not, however, in the $x$ direction, due to our neglect of viscous effects. Consequently,

$$
\left.v_{z}\right|_{z=-h}=\left.\frac{\partial \phi}{\partial z}\right|_{z=-h}=0 .
$$

At the free surface the boundary condition is specified by Bernoulli's equation

$$
\frac{\partial \phi}{\partial t}+\frac{1}{2}(\operatorname{grad} \phi)^{2}+g u=0,
$$

where $g$ is the acceleration due to gravity. The constant atmospheric pressure $p_{0}$ has been eliminated by the transformation $\phi \rightarrow \phi+(1 / \rho) p_{0} t, \rho$ being the density of the liquid.

Finally, we need the condition that the surface remains specified by the equation

$$
F(x, z, t) \stackrel{\text { def }}{=} z-u(x, t)=0 .
$$

At time $t+d t$ the displaced surface is given by
$F\left(x+v_{x}^{n} d t, z+v_{z}^{n} d t, t+d t\right)=0$, where $\nabla^{n}$ is the velocity normal to the surface.

Consequently, since $\nabla^{n} \cdot \nabla F=\nabla \cdot \nabla F$, we have as our condition

$$
\frac{d F}{d t}=\frac{\partial F}{\partial t}+v_{x} \frac{\partial F}{\partial x}+v_{z} \frac{\partial F}{\partial z}=0
$$

or in terms of $\phi$ and $u$, using (2.6)

$$
\frac{\partial \phi}{\partial z}-\frac{\partial \phi}{\partial x} \frac{\partial u}{\partial x}-\frac{\partial u}{\partial t}=0
$$

In the linear region Eq. (2.8) simply expresses that the time derivative of the position of the surface, $\partial u / \partial t$, equals the $z$ component of the velocity $\partial \phi / \partial z$ at the surface.

Solitary waves arise as a consequence of balancing the effects of nonlinearity with dispersion. One performs a simultaneous expansion in two dimensionless variables, $u_{m} / h$, where $u_{m}$ is the maximum elevation, and $h^{2} / \lambda^{2}$, where $\lambda$ is a typical horizontal length scale. In order to perform this expansion we express a solution of (2.2) satisfying the boundary condition (2.4) as a wave packet

$$
\phi(x, t)=\frac{1}{2 \pi} \int_{-\infty}^{\infty} d k \cosh k(h+z) e^{i k x} g(k, t),
$$

where the weight function $g(k, t)$ has its Fourier transform $f(x, t)$ given by

$$
f(x, t)=\frac{1}{2 \pi} \int_{-\infty}^{\infty} d k e^{i k x} g(k, t)
$$

equal to the value of $\phi$ at the bottom of the tank, $z=-h$.

The superposition (2.9) may be used to perform a gradient expansion, using the identity $i k e^{i k x}=(\partial / \partial x) e^{i k x}$, along with the assumption that $g(k, t)$ falls off sufficiently rapidly for large $k$. With the usual convention of writing a derivative $\partial \phi / \partial x$ as $\phi_{x}, \partial^{2} \phi / \partial x \partial t$ as $\phi_{x t}$, etc., we get by expanding (2.9) for $z=u$, utilizing $\cosh x \simeq 1+\frac{1}{2} x^{2}$, that

$$
\phi \simeq f-\frac{1}{2}(h+u)^{2} f_{x x}
$$

and

$$
\phi_{z}=-(h+u) f_{x x}+\frac{1}{6}(h+u)^{3} f_{x x x x} .
$$

Thus (2.8) becomes

$$
u_{t}+\left[(h+u) f_{x}\right]_{x}-\frac{1}{6} h^{3} f_{x x x x}=0
$$

by keeping terms to the given order in our expansion, while Bernoulli's equation is

$$
f_{t}+g u-\frac{1}{2} h^{2} f_{x x t}+\frac{1}{2}\left(f_{x}\right)^{2}=0 .
$$

When the nonlinear terms in (2.13) and (2.14) are neglected the resulting dispersion relation for propagating plane waves becomes

$$
\omega^{2}=c_{0}^{2} k^{2}\left(1-\frac{1}{3} k^{2} h^{2}\right),
$$

where $c_{0}$ is the velocity of shallow water waves,

$$
c_{0}=\sqrt{g h} \text {. }
$$

The result (2.15) is consistent with our scheme of expansion, which keeps the leading dispersive terms of relative order $h^{2} / \lambda^{2}$, where $\lambda$ is the wavelength $2 \pi / k$.

Equations (2.13) and (2.14) may be combined to one equation, the Korteweg-deVries equation, by expanding in terms of $u_{m} / h$ and $h^{2} / \lambda^{2}$, assuming a disturbance $u$, which moves in a definite direction, say along the positive $x$ axis.

To facilitate this procedure we introduce the function $w=f_{x}$, which has the dimensions of velocity, and rewrite 
Eqs. (2.13) and (2.14) after scaling all variables according to $x \rightarrow \lambda x, t \rightarrow \lambda t / c_{0}, u \rightarrow u_{m} u$, and $w \rightarrow w g u_{m} / c_{0}$, where $u_{m}$ as before denotes the maximum value of $u$ and $\lambda$ denotes the horizontal length scale. Then (2.13) and (2.14) become

$$
\begin{aligned}
& u_{t}+[(1+\alpha u) w]_{x}-\frac{1}{6} \beta w_{x x x}=0, \\
& w_{t}+u_{x}-\frac{1}{2} \beta w_{x x t}+\alpha w w_{x}=0,
\end{aligned}
$$

where $\alpha=u_{m} / h$ and $\beta=h^{2} / \lambda^{2}$. When $\alpha=\beta=0$ the linear equations have a solution, corresponding to a disturbance moving in the direction of positive $x$, given by

$$
w=u \text {, where } u_{t}+u_{x}=0 .
$$

In order to determine an equation for $u$, valid to first order in $\alpha$ and $\beta$, we write $w=u+\alpha A+\beta B$ and insert in (2.17) and (2.18). The two equations (2.17) and (2.18) become consistent (to linear order), if $A=-\frac{1}{4} u^{2}$ and $B=\frac{1}{3} u_{x x}$, and yield the Korteweg-deVries equation

$$
u_{t}+u_{x}+\frac{3}{2} \alpha u u_{x}+\frac{1}{6} \beta u_{x x x}=0 \text {. }
$$

With the time and space variables restored this equation becomes the long wave or Korteweg-deVries equation in its usual form,

$$
u_{t}+c_{0} u_{x}+\frac{3}{2} \frac{c_{0}}{h} u u_{x}+\frac{1}{6} h^{2} c_{0} u_{x x x}=0,
$$

which forms the basis for the subsequent discussion.

\section{THE SOLITARY WAVE}

Starting with Eq. (2.21) where $x$ and $t$ are laboratory space and time, $u$ is the wave height and $h$ is the depth of still water, it is convenient to transform to a moving frame for which

$$
\begin{aligned}
\Phi & =-u, \\
x^{\prime} & =\sqrt{\frac{3}{2}} h^{-3 / 2}\left(x-c_{0} t\right), \\
t^{\prime} & =\left(\sqrt{\frac{3}{2}} c_{0} h^{-5 / 2} / 4\right) t .
\end{aligned}
$$

After the primes have been dropped, one sees that $(2.21)$ takes the form of (1.1). A solitary wave solution is obtained by assuming that

$$
\Phi(x, t)=\widetilde{\Phi}(x-v t),
$$

where $v$ is an undetermined traveling wave speed. This assumption simplifies (1.1) from a partial differential equation to the ordinary differential equation

$$
\widetilde{\Phi}_{x x}=v \widetilde{\Phi}+3 \widetilde{\Phi}^{2},
$$

which is immediately recognized as a nonlinear pendulum problem which can be easily integrated to obtain (1.3).

\section{THE ASSOCIATED LINEAR PROBLEM}

Associated with the Korteweg-deVries equation

$$
\Phi_{t}-6 \Phi \Phi_{x}+\Phi_{x x x}=0
$$

are evolution equations for the linear scattering problem

$$
\begin{aligned}
& \psi_{x x}+[E-\Phi(x, t)] \psi=0 \\
& \psi_{t}=-4 \psi_{x x x}+3\left[\Phi \psi_{x}+(\Phi \psi)_{x}\right]
\end{aligned}
$$

Eigenvalues $\left(E_{n}\right)$ for solutions $\left(\psi_{n}\right)$ of this linear problem are constant. This can be seen by checking directly that

$$
\frac{d E_{n}}{d t}=0 \text {. }
$$

This surprising analytical fact was put to use in Ref. 2 as the basis of a linear method for finding the solution of (4.1) evolving from an arbitrary initial disturbance $\Phi(x, 0)$. The method proceeds as follows.

Step 1. Use $\Phi(x, 0)$ in $(4.2)$ to calculate the scattering data at $t=0$. [These data consist of three parts: (i) the bound state eigenvalues, (ii) the reflection coefficient for $E>0$, and (iii) residues of poles of the reflection coefficient at the bound states.]

Step 2. Find the evolution of these scattering data with time. (This is easy because as $x \rightarrow \pm \infty, \psi_{x x}+E \psi=0$ and $\psi_{t}+4 \psi_{x \times x}=0$.)

Step 3. From the scattering data at time $t$ perform an inverse scattering calculation to obtain $\Phi(x, t)$. (This is discussed in detail in Refs. 3-13.)

For an experimental demonstration it is sufficient to note that each bound state of $(4.2)$ corresponds to a soliton component

$$
\widetilde{\Phi}_{n}(x, t)=-\frac{1}{2} v_{n} \operatorname{sech}^{2}\left[\frac{1}{2} \sqrt{v_{n}}\left(x-v_{n} t\right)\right],
$$

where

$$
E_{n}=-v_{n} / 4
$$

is the associated bound state eigenvalue of (4.2).

Suppose $\Phi(x, 0)$ is initially the square-well potential

$$
\begin{aligned}
\Phi(x, 0) & =0 \text { for }|x|>\frac{1}{2} x_{0} \\
& =-u_{0} \text { for }|x|<\frac{1}{2} x_{0} .
\end{aligned}
$$

Then, from a standard reference on elementary quantum theory, ${ }^{19}$ the condition for finding at least $n$ bound states is

$$
u_{0} x_{0}^{2}>(n-1)^{2} \pi^{2} \text {. }
$$

Using the scaling (3.1) to translate back into the laboratory system, this condition becomes

$$
\left(\frac{u_{0}}{h}\right)\left(\frac{x_{0}}{h}\right)^{2}>(n-1)^{2} \frac{3}{2} \pi^{2} \text {. }
$$

As we shall see in the following sections, it is not difficult

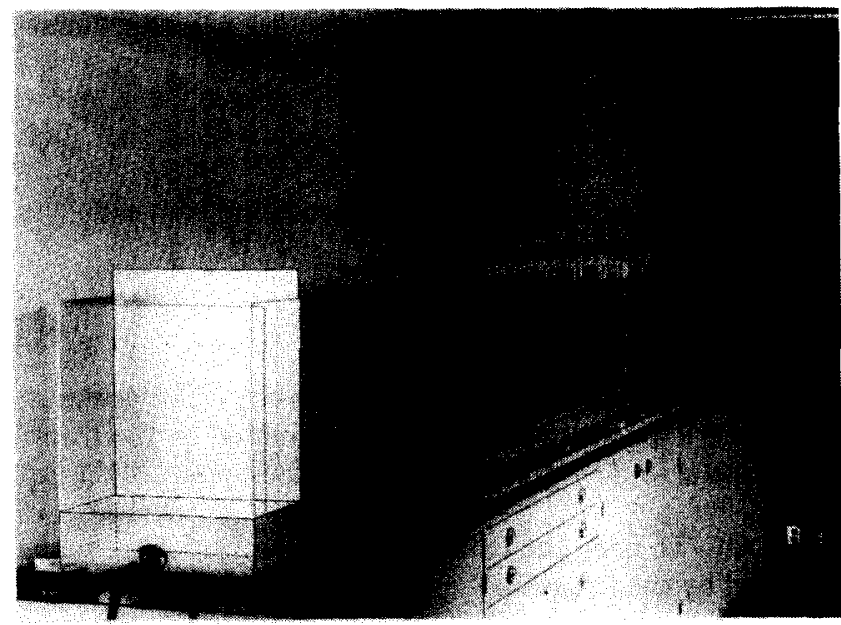

Fig. 1. The wave tank on its wood support, seen from the tap end. The step is $10 \mathrm{~cm}$ long, the level difference $5 \mathrm{~cm}$, and the basic level $5 \mathrm{~cm}$. Note the connection in the middle. 


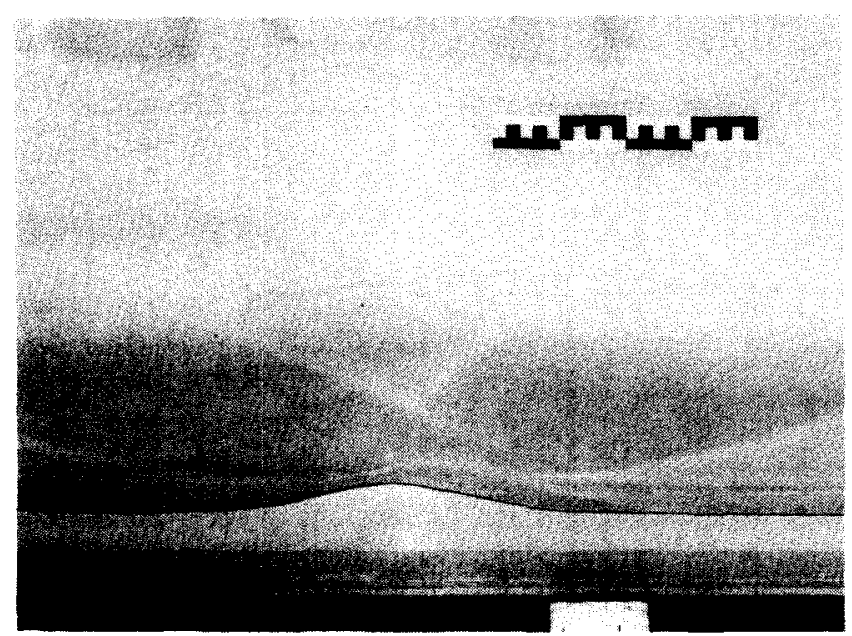

Fig. 2. A single wave going right. The scale on top is $20 \mathrm{~cm}$.

to demonstrate experimentally that this is a condition for generating at least $n$ solitons in our wave tank experiment.

\section{THE DEMONSTRATION APPARATUS}

The wave tank (see Fig. 1) is $5.5 \mathrm{~m}$ long, $0.6 \mathrm{~m}$ deep, and $0.4 \mathrm{~m}$ wide. Except for the back side, which is white (opaque) Perspex, the building material is transparent Perspex glued together. The thickness of the wall is $5 \mathrm{~mm}$, while that of the bottom is $8.5 \mathrm{~mm}$. The front and the back side of the tank are connected by a number of $5 \times 1.5 \mathrm{~cm}^{2}$ beams placed $30 \mathrm{~cm}$ apart. To be able to move the tank it is possible to disconnect it in the middle, where there is a soft rubberpacking in a 5-mm groove. The groove is constructed of two layers of Perspex plates glued on the outer surface of the tank, the inner one a little shorter than the one outside. The system is closed together by four snap locks with variable force calibration. The tank has a smooth inner surface except for the tiny grooves described below. With moderate pressure a basic water level up to $20 \mathrm{~cm}$ is possible corresponding to a total volume of 500 liters. The whole tank is on wooden bars, placed on a wooden plate, and adjusted horizontally with wedges to ensure a constant water level in the whole tank. To be able to take water out of the tank, a tap $\left(\frac{1}{2} \mathrm{in}\right.$.) is placed in one of the end surfaces near the bottom.

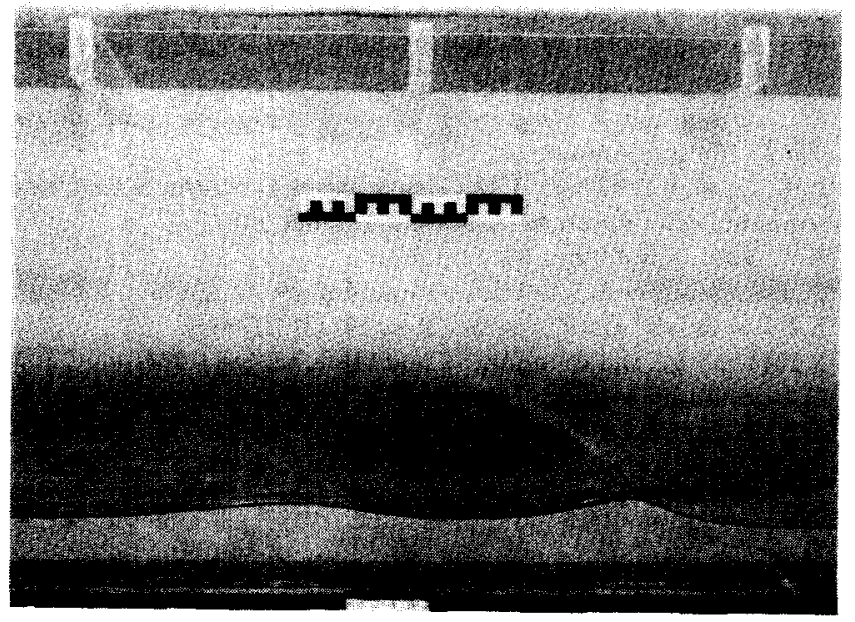

Fig. 3. A double wave going right.

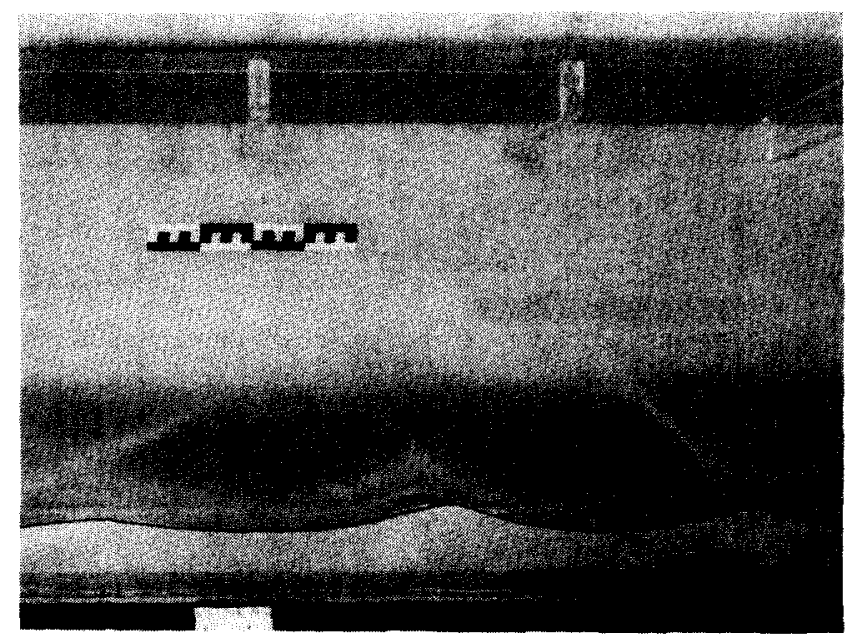

Fig. 4. A triple wave going right.

For distances $10,20,30,40,50$, and $60 \mathrm{~cm}$ from both ends vertical grooves are cut in the sides (and the bottom) to guide a 2-mm aluminum plate. The grooves are a little more than $2 \mathrm{~mm}$ wide in order to make the plates move easy. To create waves a certain amount of water is poured into the small space separated by the aluminum plate (see Fig. 1). Before filling water in the channel, a little silicone grease is filled in the grooves to keep the space defined by the plate reasonably tight.

To perform an experiment the tank and a 10-liter bucket are filled up with water colored with a small amount of fluorescein dissolved in ethanol to give a contrast from the opaque back side of the tank. The basic level in the tank is typically $5 \mathrm{~cm}$ (corresponding to a total water volume of approximately 100 liters). An aluminum plate is pushed down in one of the grooves and the level in this space is increased by $2-15 \mathrm{~cm}$ relative to the level on the other side of the plate. In our experiments this level difference was constant for several minutes. After a quick removal of the plate, the step in the small space creates one or several waves running along the tank. At the opposite end of the tank the wave is reflected, and in this way goes back and forth 10-20 times without changing its shape. Steps smaller than $2 \mathrm{~cm}$ are difficult to see, while those bigger than $15 \mathrm{~cm}$ (depending on the basic level) become turbulent. In addition, a too substantial amount of water adds to the basic level and disturbs the experiment. To keep a constant basic level the tap is used between each experiment.

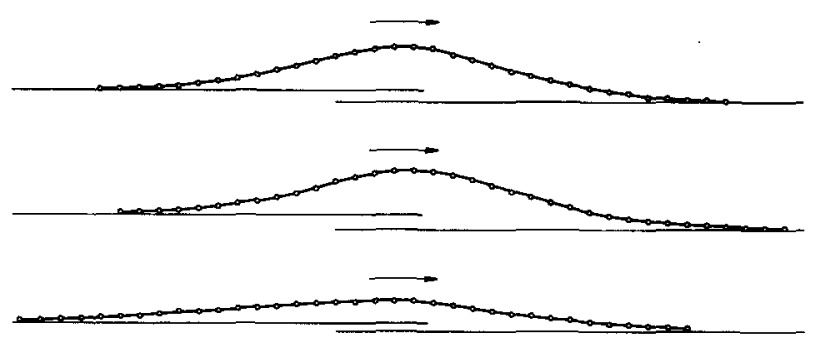

Fig. 5. Three different single waves going right. The solid curves are experimental, the dots obtained from theory, as explained in the text. Note that the basic level in the drawings (and calculations) is different on the two sides because of the water flow coming from the step. 

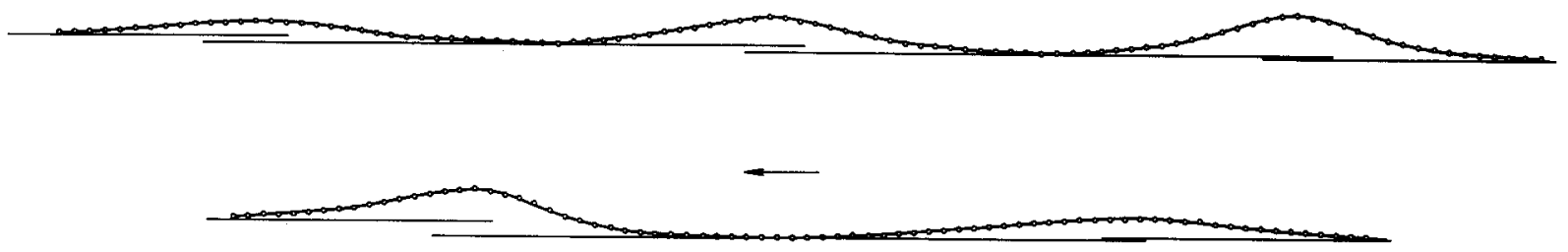

Fig. 6. A triple and double wave, the first going right, the second after reflection going left. The solid curves are experimental, the dots obtained from theory, as explained in the text.

\section{RESULTS}

For the chosen basic level we use the condition (4.9) to choose the height of the step, $u_{0}$, corresponding to the possible values of the step width $x_{0}=(10,20, \ldots, 60 \mathrm{~cm})$. Only results for $2 \mathrm{~cm}<u_{0}<12 \mathrm{~cm}$ are used, as discussed in Sec. V. If the basic level $h=5 \mathrm{~cm}$, the parameters $\left(x_{0}, u_{0}\right)$ could according to (4.9) be $n=1:(10,8.2), \quad n=2:(20,8.2)$, $n=3:(30,4.6), n=4:(40,5.3)$, and $n=5:(50,5.7)$. With these choices the total amounts of water are kept at a reasonable level. To demonstrate that the same number of waves are created in several different ways we use for $n=2$ the three sets of parameters $(20,8.2),(30,3.7)$, and $(40,2.1)$. The experiments fully support the condition (4.9), as the number of waves are in accordance with this criterion. For high amplitudes new waves are now and then created at the reflection from the end, otherwise the shape of waves is not disturbed by the reflection. In some of the experiments, the expected number of waves only developed after a few meters of propagation. Experiments for $h=3.5 \mathrm{~cm}$ to $h=10$ $\mathrm{cm}$ were tried out with results in accordance with theory. Waves for $h=5 \mathrm{~cm}$ are seen on the photos of Figs. 2-4.

It is apparent from the photos that the shape of the solitary waves is similar. By measuring the velocity of the waves (by a stopwatch) as a function of the amplitude, $u$, and level, $h$, it is possible to calculate the shape from the formula (4.5) and then draw the theoretical curves on Figs. 5 and 6 . The solid curves on Figs. 5 and 6 are drawn from photographs, the points are calculated from the theory. As seen on the drawing the basic levels are different on the two sides of the top, because of the amount of water flowing from the "step." To calculate the theoretical points we use the basic level, the maximum amplitude, and the measured velocity. No fitting parameters are used. The points are in this way calculated in two parts from the middle.

The velocity is found to exhibit a dependence on $h$ given by $c_{0}=\sqrt{0.89 g h}$. The calculation of the shape is sensitive to the numerical factor. A $2 \%$ increase in this factor could be easily distinguished in the drawings on Figs. 5 and 6. The different surface levels, as indicated on Figs. 5 and 6 , are also easily detectable, down to a difference of $1 \mathrm{~mm}$. The consistency between theory and experiment is generally excellent for a demonstration experiment.

\section{CONCLUSION}

We have seen how one may illustrate some important features of nonlinear wave propagation in a water tank experiment. The simple relation between the number of soli- tary waves and the linear dimensions of the step and the basic water level is convincingly demonstrated in our experiment. The characteristic shape of a soliton and its remarkable stability is also brought out very well. The demonstration apparatus is simple to construct, reliable, and can be used for a number of other wave experiments.

As a practical illustration of the powerful inverse scattering method, such a water tank experiment is of interest to students of both mathematics and physics. As a lecture room demonstration, the experiment described in this paper connects the relation between soliton number and geometry with a linear eigenvalue problem well known from elementary quantum mechanics, and it gives the student some appreciation of the peculiar properties of propagating, reflecting, and interacting solitary waves.

a) On leave from Center for Nonlinear Studies, Los Alamos National Laboratory, Los Alamos, NM 87545.

'N. J. Zabusky and M. D. Kruskal, Phys. Rev. Lett. 15, 240 (1965).

${ }^{2}$ C. S. Gardner, J. M. Greene, M. D. Kruskal, and R. M. Miura, Phys. Rev. Lett. 19, 1095 (1967).

${ }^{3}$ Solitons in Action, edited by K. Lonngren and A. C. Scott (Academic, New York, 1978).

${ }^{4}$ Solitons and Condensed Matter Physics, edited by A. R. Bishop and T. Schneider (Springer-Verlag, Berlin, 1978).

${ }^{5}$ F. Calogero, Nonlinear Evolution Equations Solvable by the Spectral Transform (Pitman, London, 1978).

${ }^{6}$ V. E. Zakharov, S. V. Manakov, S. P. Novikov, and L. P. Pitayevsky, Theory of Solitons. The Method of the Inverse Scattering Problem (Nauka, Moscow, 1980) (in Russian).

${ }^{7}$ G. L. Lamb, Elements of Soliton Theory (Wiley, New York, 1980).

${ }^{8}$ Solitons, edited by R. K. Bullough and P. J. Caudrey (Springer-Verlag, Berlin, 1980).

${ }^{9} \mathrm{G}$. Eilenberger, Solitons (Springer-Verlag, Berlin, 1981).

${ }^{10} \mathrm{~W}$. Eckhaus and A. V. Harten, The Inverse Scattering Transformation and the Theory of Solitons (North-Holland, Amsterdam, 1981).

${ }^{11} \mathrm{M}$. J. Ablowitz and H. Segur, Solitons and the Inverse Scattering Transform (SIAM, Philadelphia, 1981).

${ }^{12}$ F. Calogero and A. Degasperis, Spectral Transform and Solitons (North-Holland, Amsterdam, 1982).

${ }^{13}$ R. K. Dodd, J. C. Eilbeck, J. D. Gibbon, and H. C. Morris, Solitons and Nonlinear Wave Equations (Academic, London, 1982).

${ }^{14}$ A. C. Scott, Am. J. Phys. 37, 52 (1969).

${ }^{15}$ D. J. Korteweg and G. deVries, Philos. Mag. 39, 422 (1895).

${ }^{16}$ J. S. Russell, Br. Assoc. Adv. Sci. Rep. 14, 331 (1844).

${ }^{17}$ J. L. Hammack and H. Segur, J. Fluid Mech. 65, 289 (1974).

${ }^{18}$ G. B. Whitham, Linear and Non-linear Waves (Wiley, New York, 1974).

${ }^{19}$ See, for example L. I. Schiff, Quantum Mechanics (McGraw-Hill, New York, 1968), 3rd ed., pp. 39-42; or L. D. Landau and E. M. Lifshitz, Quantum Mechanics (Pergamon, Oxford, 1965), pp. 63-67. 